\title{
Potenciales beneficios y riesgos de sistemas mini- redes híbridos fotovoltaicos
}

\author{
Pajuelo Valerio Martin ${ }^{1}$, Pajuelo Acuña Flavia² \\ ${ }^{1}$ Pos Grado FIIS, Universidad Nacional de Ingeniería., Av. Túpac Amaru 108, Lima, Perú \\ ${ }^{2} \mathrm{FH}$, Universidad de Lima, Av. Javier Prado este 4600, Lima, Perú \\ E-mail: martinpajuelo@yahoo.com,20161061@aloe.ulima.edu.pe
}

DOI: https://doi.org/10.33017/RevECIPeru2016.0011/

\section{Resumen}

Sistemas mini-redes híbridas fotovoltaicas (PVHMS) juegan un rol importante para facilitar la electrificación rural en los países en desarrollo, sin embargo, estos sistemas aún enfrentan importantes barreras de adopción. La tecnología ocupa un aspecto intermedio de opciones de electrificación, entre la extensión de la red tradicional y sistemas autónomos individuales, que posee elementos de cada uno pero también sus propias características distintivas. Teniendo en cuenta ello y su aplicación relativamente limitado hasta la fecha, estos sistemas son el foco de una creciente revisión de literatura. Este trabajo ha puesto de relieve una gama de posibles beneficios y riesgos asociados con la tecnología. Sin embargo, todavía no ha habido una revisión exhaustiva de estos beneficios y riesgos documentados; una comprensión de lo que es crucial para la implementación de proyectos de inversión basado en toma de decisiones. Este artículo presenta una revisión preliminar de la literatura existente para identificar los beneficios y riesgos declarados y demostrados. Los beneficios comúnmente identificados son aquellos fáciles de medir: reducción de costos y mejora de los servicios de electricidad. Otros beneficios tales como el social y ambiental son comúnmente menos comunes de demostrar, pero son frecuentemente declarados La mayoría de riesgos identificados incluyen tamaños de sistemas incorrectos, debido a la carga incierta, cambios relacionados a la comunidad de la zona de influencia, estándares y compatibilidad de equipos, modelos de negocios inapropiados y riesgos relacionados con el aislamiento geográfico

\section{Descriptores: Fotovoltaico, Hibrido, Mini-red, Riesgo, Beneficio, Electrificación Rural}

\begin{abstract}
Photovoltaic hybrid mini-grid systems (PVHMS) are expected to play a major role in facilitating rural electrification in the developing world, however these systems still face significant barriers to adoption. The technology occupies a middle ground of electrification options e between traditional network extension and individual home systems, possessing elements of each yet also their own distinctive Characteristics. Given this, and their relatively limited application to date, such systems are the focus of a growing body of literature. This work has highlighted a range of potential benefits and risks associated with the technology. However, there still has not been a comprehensive review of these documented benefits and risks; an understanding of which is crucial for informed project investment and implementation decision-making. This paper presents a preliminary review of the existing literature to identify claimed and demonstrated benefits and risks. The most commonly identified benefits are those that are easy to measure: reduced cost and provision of improved electrical services. Other benefits such as the social or environmental benefits are less commonly demonstrated, but are frequently claimed. The major risks identified included incorrect system sizing due to load uncertainty, challenges related to community integration, equipment compatibility issues, inappropriate business models and risks associated with geographical isolation. For all of these types of risks, associated mitigation strategies were also identified in the literature. Further research including industry surveys and additional case studies will be required to validate what has been observed in the literature to date, and
\end{abstract}


identify progress as the technology matures, costs fall and stakeholders learn from these previous experiences.

Keywords: Photovoltaic, Hybrid, Mini-grid, Risks, Benefits, Rural electrification.

\section{Introducción}

El impacto potencial de mini-redes para la prestación de electrificación rural ha sido comparado con el impacto revolucionario de la tecnología inalámbrica y móvil en servicios de telecomunicaciones en los países en desarrollo [1]. Grandes redes de postes fijos y cableado utilizados para conectar a los proveedores de servicios de telecomunicaciones centralizado, a sus clientes empiezan a mirar redundante como más ágiles retos geográficos de redes celulares lo supera rápidamente y a bajo costo, con el fin de satisfacer la demanda donde y cuando se necesite.

Se ha logrado progresos considerables en las últimas décadas para extender las redes principales dentro de los países en desarrollo para alcanzar más cobertura en la población rural. Sin embargo, el número de personas sin acceso a servicios modernos de energía todavía se estima en alrededor de 1.4 billones [2]. Aquellos que permanecen desconectadas están en localidades que son muy difíciles o costosos servir a través de extensión de la red existente.

En segundo lugar, enfoques distribuidos para la prestación de servicios de electricidad, incluyendo mini-redes y sistemas independientes, al igual que las comunicaciones inalámbricas, ofrecen oportunidades para nuevas tecnologías y nuevos mercados [3]. Para las comunidades rurales remotas, esto podría significar acceso a electricidad donde de otro modo sería inviable económicamente o técnicamente, o han tomado décadas para lograr [4].

Si los programas rurales electrificación pueden ser diseñados e implementado efectivamente, beneficios adicionales para las comunidades podrían incluir una conexión a la red más confiable, un menor coste servicio e impulsar la demanda, en lugar de abastecimiento, con posibles beneficios añadidos de desarrollo económico local, puestos de trabajo y formación [5,6]. Mini-redes podrían desempeñar un papel importante de energía en comunidades que están demasiado alejadas para ser conectada a la red principal, pero cuyo servicio de energía necesita disposición son más allá de las capacidades de sistemas solares domésticos individuales, y donde hay una oportunidad de recursos agregados en equipos para financiar múltiples actividades en función a la energía.

Parece que mini-redes aún no han alcanzado su potencial de electrificación rural. Mientras Solar Home Systems (SHS) ahora han alcanzado mayor y creciente despliegue [7] Tecnología de mini-redes ha luchado para escalar y cumplir su potencial; estimado por la IEA que más del $40 \%$ de la nueva generación de energía eléctrica necesaria para proporcionar acceso universal a los servicios energéticos modernos entre 2010 y 2030 en el mundo [2]. Una serie de barreras substanciales a escala ha sido identificada en la literatura $[8,9]$. Miniredes autónomas comúnmente dependen de suministro de combustible diésel. El alto precio del combustible diésel, que es volátil y tendencias [10]; y la logística asociada al transporte y distribución que incrementa sustancialmente su costo en ubicaciones remotas es una importante carga de utilidades hacia los consumidores, así como los gobiernos que subvencionan el diésel en muchos países.

Los costos de combustible y las características operacionales de sistemas diésel a menudo corren el riesgo de trabajar para sólo un cierto número de horas al día. Existe algunos avances o de un camino medio en una mini-red con generación de RES combinados con sistemas diésel convencional (referidos a como un mini-redes híbridas) ha sido ampliamente identificada como un método para reducir el consumo de combustible, lograr la carga, generación de escala y diversidad evitando los altos costos y molestias del almacenaje en bancos de baterías [11-14]. PV es menor el costo y más apropiado que el viento en aplicaciones remotas de mini-redes, como las turbinas de viento se convierten en menos rentables cuanto más pequeños y el recurso viento es espacialmente variable y es más difícil de estimar que el recurso solar.

Información reciente hace referencias, importantes sobre la reducción en el precio de los módulos fotovoltaicos en los últimos cinco años, han resultado costos levados de generación fotovoltaica PV comparado con la generación diésel para producir electricidad, para muchas aplicaciones de mini-redes [11]. De hecho, PVHMS ahora se 
encuentran con frecuencia a ser la opción de menos costo para satisfacer la necesidad de electrificación rural [12]. Hay una amplia variedad de sistemas PV híbrido mini-redes (PVHMS) sistema configurados y los niveles de penetración de PV que caen dentro de esta categoría [15,16]. Werner y Breyer [17] han completado una revisión exhaustiva de las configuraciones de sistemas instalados según lo documentado en la literatura.

Esta investigación por lo tanto, se centra en PVHMS $y$, en particular, la pregunta ¿por qué no hemos realizado un más amplio despliegue de estas miniredes hasta la fecha? El foco de nuestro análisis está en el inter-relación de beneficios y riesgos asociados con la implementación de sistemas. En particular, hasta la fecha no se ha considerado sistemáticamente todos los beneficios potenciales y acompañando a los riesgos asociados con PVHMS. Este trabajo pretende abordar esta brecha existente en la literatura y compilar una revisión cualitativa de riesgos conocidos y beneficios en PVHMS utilizando como un primer paso para comprender mejor el perfil de riesgo/beneficio.

\section{Definición de propiedad, beneficio y riesgo}

La identificación y evaluación de riesgos y beneficios es vital en cualquier toma de decisiones. Beneficios y riesgos se asocian a diferentes partes involucradas en una decisión y por lo tanto se consideran diferenciados dependiendo del papel y las responsabilidades del proceso. Por ejemplo en el caso de electrificación rural, mientras que los diseñadores de sistemas podrían centrarse en los aspectos técnicos, financieros, el propósito potencial podría ver los riesgos y beneficios económicos 0 políticos. El propósito de esta investigación, es evaluarlos desde una perspectiva de propietarios de los sistemas, como esto nos dará el punto de vista más completa.

Para este artículo, propietario será definido como la entidad que inicia el proyecto y financia su entrega (o en muchas aplicaciones de PVHMS, facilita el financiamiento a través de fondos donados). Otras interpretaciones de la propiedad son los responsables de la operación y mantenimiento del sistema, que no es apropiado para el caso de PVHMS, puesto que este papel es a menudo subcontrata a terceros [12]; o por lo general, la entidad que recibe beneficios del proyecto [árbitros para mostrar es común]. Este último también a menudo no es el caso de PVHMS, por ejemplo, una utilidad de gobierno puede poseer un sistema operarlo pero a un costo de ruptura incluso (o tal vez hacer una pérdida), y los beneficiarios son, en cambio, los usuarios finales

Los propietarios de PVHMS pueden ser organizaciones públicas o privadas, buscando acceso a nuevos mercados, 0 acusado de proporcionar electricidad confiable servicio en comunidades rurales. Se puede recibir apoyo de donantes internacionales hacia el suministro de energía rural y puede confiar en los desarrolladores de proyectos separados o las organizaciones no gubernamentales para la implementación. Un factor de gran éxito para todos los proyectos es poseer claridad sobre la propiedad y las responsabilidades de cada interesado durante toda la vida del proyecto (como se destaca en Ref. [18] para el caso de SHS). Dependiendo de las metas y responsabilidades contractuales del propietario, se acumulen beneficios y los riesgos a una gama de diferentes partes interesadas. Las partes interesadas más importantes deben, por supuesto, las comunidades que estos sistemas están destinados a servir. Buena alineación de incentivos, o los costos/beneficios y riesgos por las comunidades y los que toman la decisión es probable que los conduzca a los mejores resultados.

Para los propósitos de este artículo, beneficios son definidos como ventaja adquirida a través de la elección de PVHMS en comparación con otras opciones técnicas. Los riesgos son considerados en el sentido del proyecto, es decir, incertidumbre, o el riesgo de un acontecimiento imprevisto o actividad que podría impactar los resultados o avances del proyecto de una manera positiva o negativa. Los riesgos y beneficios deben considerarse juntos, ya que si los beneficios aumentan entonces inevitablemente aumentará el apetito por el riesgo de las decisiones. En el caso de tecnología de energías renovables la falta de comprender y viabilizar la percepción de riesgo y beneficios han sido identificados como una barrera de adopción [19,20]. Proyectos financiados para energías renovables requieren un significativo capital en comparación con proyectos que financian generación diésel. Proyectos de PVHMS son considerados de mayores riesgos debido a la complejidad de la tecnología y la experiencia limitada en comparación con proyectos de generación diésel tradicional [21]. Además aplicaciones de tecnología en comunidades remotas de países en desarrollo requiere técnicas adicionales, cambios institucionales y económicos

\section{Metodología}

Una revisión de literatura se basó en trabajos seleccionados de bases de datos académicos, 
informes en línea, actas de la Conferencia más grande de la industria centrada en PVHMS - La Conferencia europea en PV híbridos y Mini-redes, y la Agencia Internacional de energía (IEA) sistemas de energía fotovoltaica (PVPS) tarea 11 en PV sistemas híbridos dentro de Mini-redes, observó que trabajo en PVHMS puede ser libremente categorizadas Fig. 1. Visualización del método de revisión: papeles primera categorizadas en grupos y luego revisados para compilar beneficios/riesgos. Los resultados se clasifican en tres grupos: los más comúnmente citan beneficios de PVHMS en la literatura 1- Simulaciones técnicas (sistemas de control, almacenamiento alternativo) 2-Proyecto simulaciones (modelado, PV penetración económica) 3- Experiencia operativa (estudios de caso, las lecciones aprendidas)

Fig.1.

Visualización de revisión de método: Artículos categorizados en grupos de revisión compilada beneficio/ riesgo

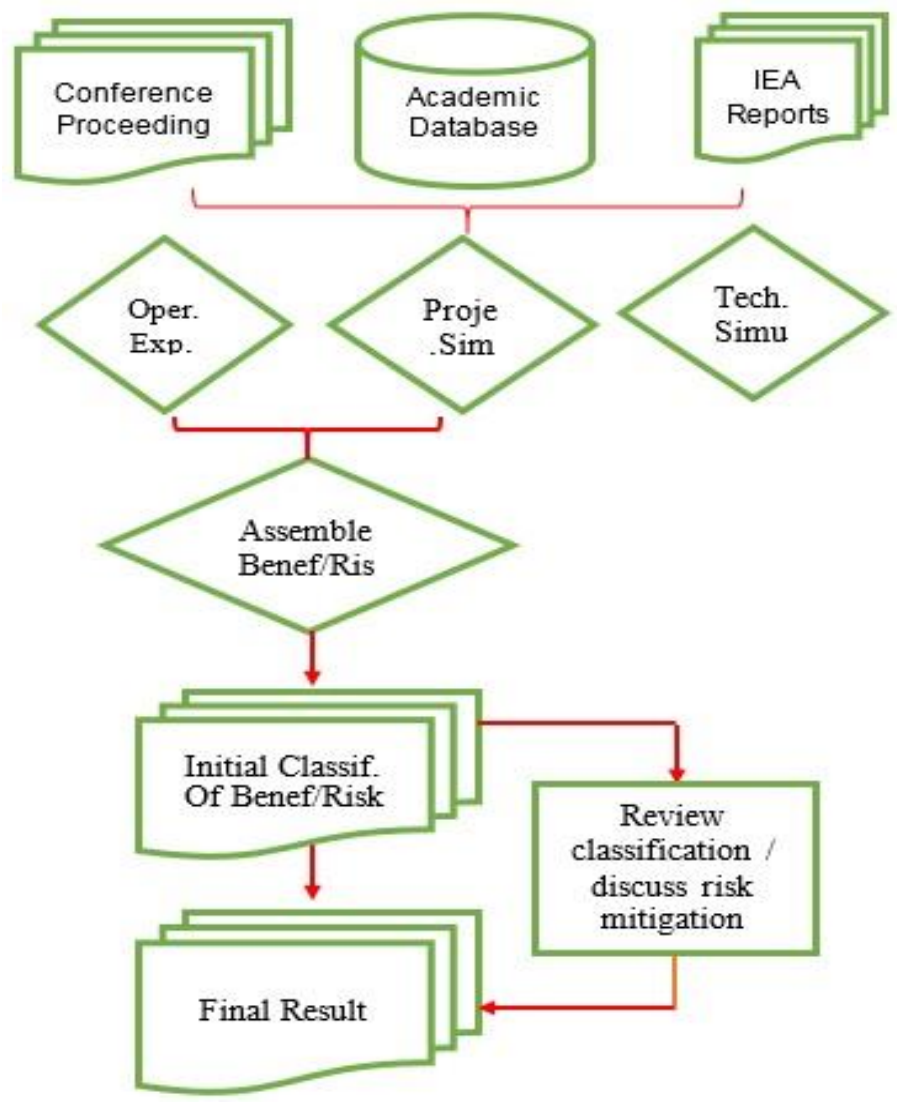

Esta revisión se centra en documentos de proyecto de simulación y experiencia operacional más relevantes para la implementación del proyecto y propiedad del sistema, mientras que las simulaciones técnicas a menudo se centran en pruebas específicas, a menudo pre mercado tecnologías o novela estrategias de funcionamiento. Se revisaron trabajos para identificar riesgos y beneficios. Se hizo una clara distinción entre esos estudios que simplemente mencionan beneficios/riesgos particulares.

Tabla 1: Beneficios

\begin{tabular}{|c|c|c|}
\hline Categoría & Beneficios & Descripción/ Demostrado \\
\hline $\begin{array}{l}\text { Tecnica } \\
\text { operación }\end{array}$ & $\begin{array}{l}\text { Implementar } \\
\text { servicios } \\
\text { eléctricos }\end{array}$ & $\begin{array}{l}\text { PVHMS puede } \text { ofrecer } \\
\text { mejores servicios personas } \\
\text { en } 20 \text { meses } \\
122,14,28-35]\end{array}$ \\
\hline Financiero & $\begin{array}{l}\text { Implementa } \\
\text { el LCOE para } \\
\text { los } \\
\text { operadores }\end{array}$ & $\begin{array}{l}\text { El nivel del costo de la } \\
\text { electricidad para mini-red } \\
\text { es más bajo que la red } \\
\text { comercial }[14,31,35,36 \text {, } \\
38,12,34,37] \text {. }\end{array}$ \\
\hline Social & $\begin{array}{l}\text { Oportunidade } \\
\text { s para } \\
\text { emprender } \\
\text { comercios } \\
\text { rurales }\end{array}$ & $\begin{array}{l}\text { Oportunidades para poder } \\
\text { emprender unidades de } \\
\text { negocios basados en } \\
\text { trabajo comunitario }[33,9 \text {, } \\
12,39] \text {. }\end{array}$ \\
\hline Ambiental & $\begin{array}{l}\text { Proteccion } \\
\text { ambiental }\end{array}$ & $\begin{array}{l}\text { Reducir la combustión de } \\
\text { diésel sistemas híbridos } \\
\text { reduce la emisión de gases } \\
\text { de efecto invernadero [28, } \\
36,32,39,46] \text {. }\end{array}$ \\
\hline
\end{tabular}

Tabla 2: Riesgos

\begin{tabular}{|c|c|c|}
\hline Categoría & Riego & Descripción/ Demostrado \\
\hline Técnica & $\begin{array}{l}\text { Calcular la } \\
\text { carga }\end{array}$ & $\begin{array}{l}\text { Como una alternativa } \\
\text { descentralizada falta de } \\
\text { estimación del tamaño de } \\
\text { carga }[42,45,48-50,9,12 \text {, } \\
\begin{array}{l}14,21,22,26,43,47,50- \\
54] .\end{array}\end{array}$ \\
\hline Organizac. & $\begin{array}{c}\text { Modelo de } \\
\text { negocios } \\
\text { inadecuado }\end{array}$ & $\begin{array}{l}\text { Modelo de negocios } \\
\text { efectivos son requeridos } \\
\text { para incrementar el } \\
\text { desarrollo y pueden ser } \\
\text { ajustados en orden de la } \\
\text { escala [41, 68, 21, 25, 53, } \\
69,64]\end{array}$ \\
\hline Social & $\begin{array}{l}\text { Integración } \\
\text { socio } \\
\text { comunitaria }\end{array}$ & $\begin{array}{l}\text { Unión de comunidades para } \\
\text { un sistema mini-red para } \\
\text { suministrar agua } \\
\text { planificación-cooperar }[22 \text {, } \\
40,71,12,14,30,51,53 \text {, } \\
25,44]\end{array}$ \\
\hline Sostenible & $\begin{array}{lr}\text { Costo } & y \\
\text { suministro de } \\
\text { diésel }\end{array}$ & $\begin{array}{l}\begin{array}{l}\text { Siempre que } \\
\text { reducción }\end{array} \\
\begin{array}{r}\text { existe } \\
\text { de }\end{array} \\
\text { requerimientos de diésel se } \\
\text { reduce el riesgo } \\
\text { particularmente el PV ha } \\
\text { bajado }[12,21,43,46,72]\end{array}$ \\
\hline
\end{tabular}




\begin{tabular}{|l|l|l|}
\hline Finanzas & $\begin{array}{l}\text { Precio } \\
\text { pagos } \\
\text { apropiados }\end{array}$ & $\begin{array}{l}\text { Los clientes son personas } \\
\text { usualmente pobres y } \\
\text { requieren subsidios para } \\
\text { acceder a la energía [73, } \\
12,30,43,53]\end{array}$ \\
\end{tabular}

Los ejemplos de la literatura son de particular interés, dado que muchos debaten los beneficios y los riesgos atribuidos a la tecnología y no proporcionan mucha orientación sobre el alcance de estos beneficios o riesgos y cómo podría abordarse. En la tabla 2 acompañando mitigaciones también han sido incluidos (Fig. 1).

\section{Resultados}

Beneficios (Tabla 1) y riesgos (Tabla 2) y han sido agrupados en tipos, con un resumen de lo que se ha escrito sobre cada uno en la literatura.

\section{Conclusiones}

La tecnología es el foco de un creciente estudio de la literatura, no ha habido ninguna revisión integral de los riesgos, cruciales para la implementación del proyecto y toma de decisiones de inversión y beneficios documentados. Este artículo ha proporcionado un examen preliminar basado en la literatura existente. Los más comúnmente identifican beneficios son aquellos que son fáciles de medir: reducción de costos y provisión de servicios eléctricos mejorados. Otros beneficios tales como los beneficios sociales 0 medioambientales se demuestran menos comúnmente, pero se menciona con frecuencia.

Los principales riesgos identificados incluyen un dimensionamiento incorrecto de sistemas debido a la incertidumbre de la carga, retos relacionados con la integración de la comunidad, problemas de compatibilidad de equipos, modelos de negocios inadecuados $y$ los riesgos asociados con el aislamiento geográfico. Para todos estos tipos de riesgos, factores atenuantes asociados fueron también identifican en la literatura, pero riesgos y mitigación apropiados son a menudo específicos del contexto. La investigación adicional se ha propuesto que validen la fecha de lo que se ha observado en la literatura con las opiniones de la industria y estudios de casos.

\section{Agradecimientos}

Al Instituto de Investigación de la FIIS de la UNI y a la Universidad de Lima.

\section{Referencias}

[1] F. Poulin, Smarter mini-grid systems and business models. In: 6th European conference on PV-hybrids and mini-grids 2012. p. 455

[2] OECD/IEA. Energy poverty e how to make modern energy access universal; 2010.

[3] M. Wollny, The off-grid market e a land of opportunities. In: 6th European conference on PV-hybrids and mini-grids 2012.

[4] ESMAP. Mini-grid design manual; 2000. Washington D.C, USA.

[5] G. Wilkins, Technology transfer for renewable energy e overcoming barriers in developing countries. London, UK: Earthscan Publications Ltd.; 2002.

[6] AB. Lovins, Small is profitable: the hidden economic benefits of distributed generation (and other distributed resources). In: Australian eco generation conference 2002.

[7] G. Shakti, Solar home system. Webpage Article [Online]. Available:

http://www.gshakti.org/index.php?option $1 / 4 \mathrm{C}$ om_content\&view $1 / 4$ article\&id $1 / 458 \&$ Itemid $^{1} / 4$ 62; 2013 [accessed12.06.13].

[8] Clean Energy Ministerial. Clean energy ministerial e roundtable 6: mini-grid development; 2013. New Delhi, India

[9] R. Van Leeuwen, The role of hybrid renewable mini-grids in providing energy access. Webinar; 2013

[10] C. Breyer, A. Gerlach, D. Schäfer, J. Schmid Fuel-parity: new very large and

Sustainable market segments for PV systems; 2010. pp. 18e22.

[12] ARE. Hybrid mini-grids for rural electrification: lessons learned; 2011.

[13] K. Mauch, Review of IEA PVPS task 11-PV hybrid systems within minigrids. In: 6th European conference on PV-hybrids and mini-grids 2012. p. 23-5.

[14] G. Léna, Rural electrification with PV hybrid systems: overview and recommendation for further deployment; 2013. 
[15] SJ. Phillips, R. Lutchman, A. Mitchell, WL. James, E. Paolinelli, Control issues and solutions in high renewable penetration on grid systems. In: 6th European conference on PV-hybrids and mini-grids 2012.

[16] L. Arribas, I. Cruz, W. Meike, High power PV-hybrid systems: is it their time now? In: 6th European conference on PV-hybrids and mini-grids 2012. №. 34.

[17] Werner C, Breyer C. Analysis of minigrids installations: an overview of system configurations. In: 27th European photovoltaic solar energy conference 2012. №. September.

[18] IEA-PVPS T9:02. Summary of models for the implementation of photovoltaic solar home systems in developing countries e part 2: practical experience;

[19] J. Painuly, Barriers to renewable energy penetration; a framework for analysis. Renew Energy Sep. 2001; 24(1):73-89.

[20] O. Waissbein, Y. Glemarec, Derisking renewable energy investment (UNDP Report); 2013.

[21] D. Turcotte, F. Sheriff, PV horizon: workshop on photovoltaic hybrid systems e summary and conclusions of the workshop; 2001. Varennes/Canada.

[22] C-W. Shyu, End users' experiences with electricity supply from standalone mini-grid solar PV power stations in rural areas of Western China. In: $6^{\text {th }}$ European conference on PV-hybrids and mini-grids 2012.

[23] X. Vallvé, H. Haraji, EA. Jaoudeh, Obeid J. Novel example of PV and WTG back up power systems for weak rural grids in Lebanon. In: 6th European conference on PV-hybrids and mini-grids 2012.

[24] PO. Moix, N. Zuchuat, A minigrid of indivual solar home systems: a distributed minigrid, concept and test site in Switzerland. In: 6th European conference on PV-hybrids and mini-grids 2012

[25] CLS. Vieira, Operation and maintenance of PV hybrid systems in remote villages in Amazonas, Brazil. In: 6th European conference on PV-hybrids and Mini-grids 2012. p. 0e3.

[26] AM. Mahmud, Analysis of the performance and impact of the rural electrification using solar hybrid system for rural schools in Sabah, Malaysia e case study. In: 6th European conference on PV-hybrids and mini-grids 2012. p. 340-7.
[27] M. Wollny, S.Tapanlis, Hybrid backup power solutions for unstable grids. In: 4th European conference on PV-hybrids and mini-grids 2008.

[28] T. Gül, Integrated analysis of hybrid systems for rural electrification in developing countries. Stockholm: Royal Institute of Technology; 2004.

[29] Alliance for Rural Electrification. Best practices of the alliance for rural electrification: what renewable energy can achieve in developing countries; 2011.

[30] X. Vallvé e WISE lecture series," WISE lecture series [Online]. Available: http: //www. youtube.com/ watch? v1/48h _ sn2Zb0l; 2012 [accessed 08.02.13].

[31] C. Breyer, C. Werner, S. Rolland, Adelmann P. Off-grid photovoltaic applications in regions of low electrification : high demand, fast financial amortization and large market potential. In: 26th European photovoltaic solar energy conference 2011. No. September.

[32] E. Macías, P. Llamas, Opportunities and challenges of rural electrification with hybrid systems. In: 4th European conference on PV-hybrids and Mini-grids 2008.

[33] A. Schroeter A, S. Martin, Profitable and affordable energy services for remote areas in Lao PDR: private e public partnership as mutual leverage for hybrid village grids in areas off the national grid Andy Schroeter. In: 4th European conference on PV-hybrids and mini-grids 2008. No. May.

[34] OO. Stephen, L. Yanli, Z. Wei, Z. Fuhong, S. Hui, Reliability analysis of switched stand-alone hybrid PV-diesel generation power system for rural electrification in Nigeria, vol. 4; 2012. pp. 663 e 676. no. 4

[35] M. Gorn, PV-hybridization of diesel electricity generators: conditions of profitability and examples in different power and storage size ranges. In: $5^{\text {th }}$ European conference on PV-hybrids and mini-grids 2010. p. 25 e 28.

[36] J. Dekker, M. Nthontho, S. Chowdhury, SP. Chowdhury. Economic analysis of $\mathrm{PV} /$ diesel hybrid power systems in different climatic zones of South Africa. Int J Electr Power Energy Syst Sep. 2012; 40 (1): 104 e 112.

[37] N. Phuangpornpitak, S. Kumar, PV hybrid systems for rural electrification in Thailand. Renew Sustain Energy Rev Sep. 2007; 
11(7):1530e43.

[49] X. Zhang, A. Kumar, Evaluating renewable energy-based rural electrification program in western China: emerging problems and possible scenarios. Renew Sustain Energy Rev Jan. 2011; 15 (1):773 e 9.

[38] AL. Schmid, CAA. Hoffmann, Replacing diesel by solar in the Amazon: shortterm economic feasibility of PV-diesel hybrid systems. Energy Policy May 2004; 32 (7):88 1 e 98.

[39] I. Ibrik I, M. Lecumberri, Techno e economic feasibility of energy supply of remote villages in palestine by $P V \quad e$ systems, diesel generators and electric grid (case studies: Emnazeil \& Atouf villages). In: 5th European conference on PV-hybrids and mini-grids 2010.

[40] P. Díaz, R. Peña, J. Muñoz, CA. D. Arias, Field analysis of solar PV based collective systems for rural electrification. Energy May 2011; 36(5): 2509 e 16.

[41] P. Konings, PV mini-grids for yap state: sustainable design for remote Pacific islands. In: 4th European conference on PV-hybrids and mini-grids 2008.

[42] F. Jochem, R. Schultz R, A. Hangula, D. Jarrett, Solar hybrid minigrid in Tsumkwe, Namibia e a holistic approach. In: 6th European conference on PV-hybrids and mini-grids 2012. p. 1 e4.

[43] G. Shrestha, C. Menke, W. Klinghammer. Case studies of PV-diesel hybrid systems in operation in rural Asia: experiences and conclusions from China, Thailand and Vietnam. In: 4th European conference on PV-hybrids and minigrids 2008

[44] W. Mohns, D. Stein, Community powerhouse: a rural electrification model for Vanuatu. In: 4th European conference on PV-hybrids and mini-grids 2008.

[45] X. Wang, X. Vallve, Solar-diesel hybrid options for the Peruvian Amazon; 2007.

[46] MA. Elhadidy, Performance evaluation of hybrid (wind/solar/diesel) power systems. Renew Energy Jul. 2002; 26(3):401- 413.

[47] M. Rothert, AS. Bukvic-Schafer, Design of PV hybrid systems: energy and power requirement e the unknown input variables. In: 6th European conference on PV-hybrids and mini-grids 2012.

[48] E. de Martel, G. Marboef, G. Schweitz, J. Alzieu, E. Seyes, From feedback on international experiences to improvements of PV-diesel hybrid power system. In: 6th European conference on PV-hybrids and mini-grids 2012.
[50] C. Nielsen, F. Fiedler, Evaluation of a micro PV-diesel hybrid system in Tanzania. In: 6th European conference on PV-hybrids and mini-grids, vol. 46; 2012. P. 3-e6.

[51] A. Graillot, M. Briganti, M. Solano-Peralta, $X$. Vallvé, 15 years of field experience with the $D$ 'daily energy allowance' concept as the basis for load control and guide for social behaviour in rural micro grids. In: 6th European conference on PV-hybrids and mini-grids 2012.

[52] S. Fulton S, S. Moola, K. Polsani, S. Reddy, A. Skumanich, Hybrid microgrid configuration and expansion analysis: an India rural electrification case study in progress. In: 6th European conference on PV-hybrids and mini-grids 2012.

[53] P. Jacquin, B. Ortiz, X. Vallve, Social, economic and organizational framework for sustainable operation of PV hybrid systems within mini-grids; 2011.

[54] P. Díaz, CA. Arias, D. Sandoval, R. Peña, Field experience on operation and repowering of PV-hybrid systems; 2011.

[55] Araki I, Kroposki B, Mayr C. Design and operational recommendations on grid connection of PV hybrid mini-grids design and operational recommendations on grid; 2011.

[56] Katiraei F, Turcotte D, Swingler A, Dignard L. Modeling and dynamic analysis of a medium penetration PV-diesel mini-grid system. In: 4th European conference on PV-hybrids and mini-grids 2008.

[57] E. Rikos, S. Tselepis, A. Neris, Stability in mini-grids with large PV penetration under weather disturbances-implementation to the power system of Kythnos. In: 4th European conference on PV-hybrids and mini-grids 2008.

[58] E. Rikos, S. Tselepis, Comparative study between distributed and centralized PV generation in island power systems under variable weather conditions. In: 5th European conference on PV-hybrids and mini-grids 2010.

[59] M. Patsalides, A. Stavrou, V. Efthimiou, GE. Georghiou. Assessing the level of harmonic distortion due to PV generation in mini grids. In: 4th European conference on PVhybrids and mini-grids 2008 
[61] Schwunk S, Ortiz B, Bopp G, Vetter M. Simulation of hybrid PV systems e concepts for technology development, system design and performance improvement. In: 4th European conference on PV-hybrids and mini-grids, vol. 49; 2008. p. 0e7.

[62] G. Bopp, A. Lippkau. World-wide overview about design and simulation tools for hybrid PV systems. In: 4th European conference on PV-hybrids and minigrids, vol. 49; 2008. No. 1.

[63] B. Ortiz B, D. Sarian, J. Wachtel. "Village solar electrification: a review of feasible solutions for demand side management. In: 6th European conference on PVhybrids and mini-grids 2012. p. 2e5.

[64] J. Walter, H. Wade, T. Jensen, B. Clay, E. Langham E. Poster presentation: a trial PV mini-grid installation for small islands. In: 4th European conference on PVhybrids and mini-grids 2008.

[65] B. Kroposki. Development of microgrid technologies and standards in the U.S. In: 4th European conference on PV-hybrids and mini-grids 2008.

[66] GJ. Stapleton. Applying lessons learned from the implementation of solar home system to the implementation of hybrid systems in developing countries. In: 6th European conference on PV-hybrids and mini-grids 2012.

[67] F. Reiners, B. Burger, O. Stalter, M. Eberlin , W. Roth, Seybold F. Power electronics for next generation village energy supply. In: 6th European conference on PVhybrids and mini-grids 2012.
[68] W. Furst. Solar hybrid school project in East Malaysia. In: 5th European conference on PV-hybrids and mini-grids 2010. p. 2e5.

[69] H-W Boehnke. Djungle power e a more remote $A C$ bus. In: 5th European conference on PV-hybrids and mini-grids 2010. p. 201e3.

[70] C. Nielsen, Solar power in East Africa: wrong and much better. BlogSpot; 2012 [Online].Available: http: // carolinenielsen . blogspot. Se/2012/10/wrong-and muchbetter.html [accessed 10.06.12].

[71] S. Ruin, An open approach to hybrid power systems including demand side management. In: 6th European conference on PV-hybrids and mini-grids 2012.

[72] P. Blechinger, K. Bognar, M. Hlusiak, C. Breyer, Energy supply in mini-grids regarding renewable energies: an optimization for Petite Martinique. In: 6th European conference on PV-hybrids and mini-grids 2012. p. 1-4.

[73] R. Kimera, R. Okou, AB. Sebitosi, KO. Awodele. A concept of dynamic pricing for rural hybrid electric power mini-grid systems for sub-Saharan Africa. In: 2012 IEEE Power Energy Soc Gen Meet Jul. 2012. p. 1 e6. 